\title{
The effectiveness and safety of TNF-alpha blockers in the treatment of early psoriatic arthritis: an Italian multicentre longitudinal observational pilot study
}

\author{
Raffaele Scarpa • Mariangela Atteno • Ennio Lubrano • \\ Giuseppe Provenzano • Salvatore D'Angelo • \\ Antonio Spadaro • Luisa Costa • Ignazio Olivieri
}

Received: 20 September 2010 /Revised: 27 December 2010 / Accepted: 3 January 2011 / Published online: 1 March 2011

(C) The Author(s) 2011. This article is published with open access at Springerlink.com

\begin{abstract}
The objective of this study is to assess the effectiveness and safety of TNF- $\alpha$ blockers in a group of early psoriatic arthritis (PsA) patients with an unsatisfactory response to previous conventional treatment consecutively enrolled in five Italian centres. A 24-week open-label trial was carried out in consecutive early PsA patients classified according to the CASPAR criteria, with unsatisfactory response to previous treatments and with a DAS28 threshold as $\geq 3.2$, seen at the outpatient clinics of each centre. Exclusion criteria were previous
\end{abstract}

R. Scarpa $(\bowtie) \cdot$ M. Atteno $\cdot$ L. Costa

Department of Clinical and Experimental Medicine,

Rheumatology Research Unit, Early Psoriatic Arthritis Clinic,

University Federico II,

via Sergio Pansini no. 5,

80131 Naples, Italy

e-mail: rscarpa@unina.it

E. Lubrano

Academic Rheumatology Unit, Department of Health Science, University of Molise,

Campobasso, Italy

G. Provenzano

Rheumatology Unit, “AO-Villa SOFIA-CTO”,

Palermo, Italy

S. D’Angelo $\cdot$ I. Olivieri

Rheumatology Department of Lucania,

S. Carlo Hospital of Potenza,

Potenza, Italy

S. D'Angelo

University of Molise,

Campobasso, Italy

\section{A. Spadaro}

Dipartimento di Clinica e Terapia Medica,

Divisione di Reumatologia, Sapienza-Università di Roma,

Rome, Italy usage of TNF- $\alpha$ blockers and a disease duration $>12$ months. The choice of any of the three TNF- $\alpha$ blockers was decided by the expert's opinion, without any restriction. Effectiveness was considered as an improvement of DAS28 at 12 and 24 weeks of treatment. Secondary endpoints were an improvement of TJC, SWJ, HAQ score and PASI score. Changes from baseline to the 12- and 24-week follow-up assessments were analysed using the Wilcoxon paired sign rank test. Twenty-nine patients (14 males, 15 females, median age 37 years, range 20-65 years) were enrolled. A statistical improvement of the DAS28 was observed at 12 and 24 weeks from baseline $(p<0.001)$. Secondary endpoints also confirmed the effectiveness of the TNF- $\alpha$ blockers in the treatment of early PsA. No severe adverse events were observed during the treatment period, and no patient withdrew from the medications. This study suggests that the TNF- $\alpha$ blockers can be effective in the management of early PsA. Further controlled studies will provide more data on this challenging topic.

Keywords Early disease - EULAR response criteria . DAS28 $\cdot$ Psoriatic arthritis $\cdot$ TNF- $\alpha$ blockers

\section{Introduction}

A large body of evidences has demonstrated that the immediate detection of inflammatory joint diseases by a rapid referral to a rheumatologist can assure a precise diagnosis and a tailored therapy, with a consistent advantage in prognosis [1]. The introduction of biologic therapies for the treatment of different forms of arthritis has profoundly modified the clinical approach to rheumatic patients. By reducing articular inflammation and pain, biologic therapies really contrast the progression of joint damage. 
Psoriatic arthritis (PsA) is a debilitating condition that leads to increased morbidity and mortality [2]. Its early detection with a rapid therapeutical intervention really reduces the risk of clinical progression [3]. Unfortunately, there are many doubts that, using a traditional clinical approach, we may achieve early detection and treatment of PsA. In contrast with rheumatoid arthritis (RA), PsA patients show a reduced articular and non-articular tenderness with a threshold of articular pain which is significantly increased [4]. On the other hand, evidences showing that traditional diseasemodifying anti-rheumatic drugs (DMARDs) are really able to block clinical progression of the disease are controversial $[5,6]$. In fact, despite the use of a DMARD, follow-up shows that more than $60 \%$ of the patients starting with a mono-oligoarthritis develop a polyarthritis, with a close relationship between number of involved joints and disease duration [5].

In order to evaluate the role of biologic agents in early PsA, we designed a multicentre longitudinal observational study (LOS) based on real clinical practice and aimed at assessing the effectiveness and safety of the TNF- $\alpha$ blockers (adalimumab, etanecept, infliximab) at present recommended and available in the Italian market.

\section{Patients and methods}

Study design

The study was designed as a longitudinal and observational, and it was carried out in five Italian centres, enrolling consecutive patients affected by early PsA and attending their follow-up visit at the PsA Early Arthritis Clinic (PsA-EAC). PsA was classified based on the CASPAR criteria [7].

Inclusion criteria were patient's age $>18$ years, disease duration $\leq 12$ months and an unsatisfactory response to previous conventional treatments carried out for at least 3 months. Unsatisfactory response was therefore deemed as DAS28 threshold as $\geq 3.2$.

All patients were previously treated with non-steroidal antiinflammatory drugs (NSAIDs) and with disease-modifying anti-rheumatic drugs (DMARDs). In particular, Salazopyrin was administered in three patients and two patients were on leflunomide; both drugs were withdrawn for intolerance. Methotrexate was administered in 22 patients and among them 11 withdrew for lack of efficacy (five patients) or for intolerance (six cases, mainly for raised liver function tests). Finally, steroids was administered in a minimal percentage $(5 / 29,17 \%)$ at a dosage $\leq 5 \mathrm{mg}$ of prednisone.

The study protocol considered a recruitment period from January 2008 until December 2008.

The exclusion criteria were previous usage of anti TNF- $\alpha$ blockers, the usage of more than $10 \mathrm{mg}$ prednisone daily, variation of dosage of NSAIDs or prednisone within 2 weeks of enrolment.

The choice of any of the three TNF- $\alpha$ blockers was decided by the expert's opinion, without any restriction. However, the association with methotrexate (up to $15 \mathrm{mg}$ weekly) was allowed based on the expert's opinion.

All patients gave their written informed consent, and the study protocol was approved by the local ethical committees.

\section{Patients}

Twenty-nine consecutive patients with early PsA, routinely attending the PsA-EAC at the secondary referral centres (14 males, 15 females, median age 37 years, range $20-65$ years; median duration of disease 8 months, range 1-12 months) joined the present study protocol.

In patients eligible for therapy with TNF- $\alpha$ blockers the tuberculin skin test and interferon gamma release assay tests were carried out to screen latent tubercular infection [8]. Patients screened positive for TB test were excluded.

After the screening, all patients were given infliximab (IFN) at dosage of $5 \mathrm{mg} / \mathrm{kg}$ every $6-8$ weeks (after the usual start up and then increasing or decreasing the dosage when warranted), etanercept (ETN) $50 \mathrm{mg}$ weekly and adalimumab (ADA) $40 \mathrm{mg}$ eow.

\section{Disease activity}

The patients were followed up at 12 weeks and at 24 weeks. Clinical and laboratory assessment were carried out at baseline (T0), at 12 weeks (T1) and at 24 weeks (T2) including: physical examination, vital signs, TJC (68 tender joints), SJC (66 swollen joints), HAQ score and PASI, ESR and CRP and VAS score on patient's global assessment of disease activity.

Moreover, the DAS28 was calculated at the beginning of the TNF- $\alpha$ blockers treatment, at 12 weeks and at 24 weeks of follow-up treatment.

\section{Assessment of response}

Effectiveness was defined as an improvement of DAS28 at 12 and 24 weeks of treatment, which was therefore considered the main endpoint. Secondary endpoints were an improvement of TJC, SWJ, HAQ score and PASI score.

\section{Statistical analysis}

Descriptive data were expressed, if not otherwise specified, as median and range. Changes from baseline to the 12- and 24week follow-up assessments were analysed using the Wilcoxon paired sign rank test for the endpoints considered. All statistical procedures were two-sided at a significance level of 0.05 . 
Table 1 Demographic, baseline clinical and therapeutic characteristics of patients

Statistical analysis was carried out using the SPSS package for Windows (version 13.0; Chicago, IL).

\section{Results}

Descriptive, clinical and functional data

Main demographic and clinical characteristics of the patients studied measured at baseline and 12 and 24 weeks are reported in Table 1 . In particular, the oligoarticular pattern of the disease was the main subset observed (15/29, $52 \%$ ). An overlap with axial involvement was observed in nine patients $(9 / 29,31 \%)$, and the axial was defined by the presence of inflammatory back pain [9]. Dactylitis and nail involvement were recorded in 6/29 (20\%), 4/29 (14\%) and 5/29 (17\%), respectively.

The PASI score was overall low (median, 0.6), while the median HAQ score at the beginning was quite high. Indeed, a remarkable improvement of HAQ was observed at 12 weeks and with another significant improvement at 24 weeks.

Finally, biologic agents were chosen as follows: ETN in $19 / 29(65.5 \%)$, ADA in $7 / 29(24.1 \%)$ and IFN in $3 / 29$ (10.4\%).

\section{Effectiveness}

At baseline, median DAS28 was 5.3 (4-6.8); at 12 weeks, it dropped to $4(2.1-6.0)$ and at 24 weeks, it was $3(0.5-5.2)$ which could be considered a value in keeping with a low disease activity status.

At 12 weeks, 16 patients $(16 / 29,55 \%)$ showed an improvement of the DAS28 $>1.2$, six patients $(6 / 29,20 \%)$ showed an improvement of DAS $28>0.6 \leq 1.2$ and seven $(7 /$ $29,24 \%$ ) had an improvement of DAS28 $<0.6$. In particular, at 12 weeks, only two patients $(2 / 29,6.9 \%)$ still showed a DAS28 $>5.1$ which reflects a high disease activity.

At 24 weeks, 24 patients $(24 / 29,82 \%)$ showed an improvement of the DAS28 $>1.2$, while one patient $(1 / 29$, $3.4 \%$ ) showed an improvement of DAS28 $>0.6 \leq 1.2$ and four $(4 / 29,14 \%)$ showed an improvement of DAS $28<0.6$. Again, at 24 weeks, one patient showed a DAS28 $\geq 5.1$, and this was considered as non-responder.

Therefore, EULAR response criteria at 24 weeks were achieved by 24 patients, four had a poor response and one was a non-responder.

However, a statistical significant improvement of the DAS28 at 12 and 24 weeks was observed in all patients. Moreover, a significant improvement of the secondary endpoints was also recorded.

In Table 2 is a summary of all data on the effectiveness.

\section{Adverse events}

No serious adverse events were observed during the 24 weeks of treatment with the three biologic medications. No cases of tuberculosis or demyelinating disease were reported during this study.
Table 2 Summarized data on the effectiveness

\begin{tabular}{lcccc}
\hline Endpoints & T0 (range) & T12 (range) & T24 (range) & $p$ \\
\hline DAS 28 & $5.3(4-6.8)$ & $4(2.1-6.0)$ & $3(0.5-5.21)$ & $<0.001$ \\
Tender joints count & $8(5-18)$ & $5(1-15)$ & $2(0-10)$ & $<0.001$ \\
Swollen joints counts & $3(1-12)$ & $1(0-4)$ & $0.5(0-4)$ & $<0.001$ \\
PASI & $0.6(0-5.2)$ & $0.3(0-4.5)$ & $0.2(0-2.1)$ & $<0.001$ \\
HAQ & $1(0.5-2.62)$ & $0.37(0.1-2.2)$ & $0.25(0-1.75)$ & $<0.001$ \\
ESR & $24(3-65)$ & $15(2-86)$ & $9(2-25)$ & $<0.001$ \\
CRP & $1.5(0-36)$ & $0.6(0-11)$ & $0.3(0-12)$ & $<0.001$ \\
Patients' VAS & $70(50-90)$ & $50(30-70)$ & $35(0-90)$ & $<0.001$ \\
\hline
\end{tabular}




\section{Discussion}

The innovation of biological therapies has transformed the treatment and care of many different forms of arthritis, including RA, ankylosing spondylitis, PsA and juvenile arthritis. The current biological therapies profoundly reduce the pain and inhibit the progression of joint damage for these chronic and debilitating diseases [10, 11].These complex therapies prevent disability, and these compounds are life-altering treatment for many patients. However, for some patients, access to these therapies is limited or nonexistent due to their cost and availability.

To date, the use of biologic therapies in patients with early PsA has not been reported in randomized controlled trial, or from real clinical practice. Moreover, conventional agents are partially effective in established PsA but, in general, trails with DMARDS have not included validated outcome measures for the different manifestations of PsA [12]. However, an early intervention could also modify the whole spectrum of psoriatic disease, including extra-articular manifestations [13].

The present study, designed as LOS in five centres, showed a preliminary data on the positive effectiveness of the TNF- $\alpha$ blockers as a drug class, as previously reported [14]. In fact, all three medications, freely chosen by the experts, demonstrated to modify significantly the disease activity. In particular, the results also showed that an early intervention positively changed the patient's function as demonstrated by the rapid improvement of the HAQ, the median HAQ score at the beginning was quite high; in fact, the median HAQ score at the beginning was quite high reflecting the activity status more than the severity of the disease.

Moreover, the PASI score was overall low which is in keeping with PsA patients attending the rheumatology outpatient clinics. Another interesting point is the disease duration of the enrolled patients which was less than 12 months and, therefore, in a real early stage of the natural course of the disease. A low disease activity status was reached at 24 weeks in the majority of the patients, as deemed by the DAS28, confirming that in a relatively short period of time, it is possible to gain a well clinical control of the disease. Moreover a DAS28 $<2.6$ was observed in nine patients ( $31 \%)$ indicating a disease remission status as previously defined [15].

The choice of DAS28 as main outcome measure was based on some evidences that it resulted as the most responsive and discriminative instrument [16] as well as it was considered as an outcome measure for the peripheral joint involvement in PsA patients [17].

Recently, the British Society for Rheumatology Biologics Register (BSRBR) published the results form the largest longitudinal observation study on PsA patients and the DAS28 (EULAR response criteria) was deemed as the response criteria to the biologic agents [15]. They showed that at 6 months of treatment, $75.8 \%$ of the population studied ( $n=$
596) were EULAR responder. Our data are slightly different with a trend toward a higher percentage $(82 \%)$ but similar to some extent. However, our sample was absolutely smaller than the BSRBR, and it was focused on early PsA patients.

Therefore, the present study needs to be confirmed in a larger sample of patients in the early stages of the disease, probably recruited from a study designed as a multicentre, international based on the real clinical practice. Alternatively, results from registries could be also useful on this intriguing topic. In fact, in our study, the concomitant stable use of MTX was allowed (but was not an inclusion criterion) and then some patients were also on MTX (11/ 29, data not shown). We did not found any differences (data not shown), and these data could be biassed by the small number of patients studied even if, to some extent, these data were already reported [18].

In some ways, this study also confirms that the response to TNF- $\alpha$ blockers in psoriatic patients is greater than that observed in RA patients, as shown by the BSRBR [19]. Also, the present study showed that the biologic agents can effectively act on both major components of the psoriatic disease, even if the skin involvement was minimal in our group.

A major point against the wide usage of TNF- $\alpha$ blockers in early stages of the disease could be the high costs of these medications. However, a cost-effectiveness study on established PsA has been published by us, confirming that even in late stage of the disease, a therapeutical intervention was effective [20].

In conclusion, the present study showed that an early intervention in PsA patients with a short disease duration is really effective and it should be the real treatment approach.

Disclosure All the authors have received funding from Abbott, Wyeth and Schering Plough to attend scientific meetings and national advisory boards.

Open Access This article is distributed under the terms of the Creative Commons Attribution Noncommercial License which permits any noncommercial use, distribution, and reproduction in any medium, provided the original author(s) and source are credited.

\section{References}

1. Emery P (1994) The Roche Rheumatology Prize Lecture. The optimal management of early rheumatoid arthritis: the key to preventing disability. Br J Rheumatol 33:765-768

2. Gladman DD (2009) Psoriatic arthritis from Wright's era until today. J Rheumatol 36(supp183):4-8

3. Scarpa R, Cuocolo A, Peluso R, Atteno M, Gisonni P, Iervolino S et al (2008) Early psoriatic arthritis. J Rheumatol 35:137-141 
4. Buskila D, Langevitz P, Gladman DD, Urowitz S (1992) Smythe HA Patients with rheumatoid arthritis are more tender than those with psoriatic arthritis. J Rheumatol 19:1115-1119

5. Jones SM, Armas JB, Cohen MG, Lovell CR, Evison G, McHugh NJ (1994) Psoriatic arthritis: outcome of disease subsets and relationship of joint disease to nail and skin disease. $\mathrm{Br} \mathrm{J}$ Rheumatol 33:834-839

6. Gladman DD, Mease PJ (2006) Towards international guides for the management of psoriatic arthritis. J Rheumatol 33:1228-1230

7. Taylor W, Gladman DD, Helliwell P, Marchesoni A, Mease P, Mielants H, The CASPAR Study Group (2006) Classification criteria for psoriatic arthritis. Development of new criteria from a large international study. Arthritis Rheum 54:2665-2673

8. Bocchino M, Matarese A, Bellofiore B, Giacomelli P, Santoro G, Balato $\mathrm{N}$ et al (2008) Performance of two commercial blood IFNgamma release assays for the detection of Mycobacterium tuberculosis infection in patient candidates for anti-TNF-alpha treatment. Eur J Clin Microbiol Infect Dis 27(10):907-913

9. Rudwaleit M, Metter A, Listing J, Sieper J, Braun J (2006) Inflammatory back pain in ankylosing spondylitis: a reassement of the clinical history for application as classification and diagnostic criteria. Arthritis Rheum 54:569-578

10. Ikeda K, Cox S, Emery P (2007) Biological therapy in early arthritisovertreatment or the the way to go? Arthritis Res Ther 9:211

11. Emery P, McInnes B, van Vollenhoven R, Kraan MC (2008) Clinical identification and treatment of a rapidly progressing disease state in patients with rheumatoid arthritis. Rheumatology 47:392-398

12. Scarpa R, Peluso R, Atteno M, Manguso F, Spanò A, Iervolino S et al (2008) The effectiveness of a traditional therapeutical approach in early psoriatic arthritis: results of a pilot randomised 6-month trial with methotrexate. Clin Rheumatol 27:823-826
13. Gelfand JM, Neimann AL, Shin DB, Wnag X, Margolis DJ, Troxel AB (2006) Risk of myocardial infarction in patients with psoriasis. JAMA 296:1735-1741

14. Gladman DD (2008) Adalimumab, etanercept and infliximab are equally effective treatments for patients with psoriatic arthritis. Nat Clin Pract Rheumatol 4:510-511

15. Saad AA, Ashcroft DM, Watson KD, Symmons DPM, Noyce PR, Hyrich KL, on behalf of the BSRBR (2010) Efficacy and safety of anti-TNF therapies in arthritis: an observational study from the British Society for Rheumatology Biologics Register. Rheumatology 49:697-705

16. Fransen J, Antoni C, Mease PJ et al (2006) Performance of response criteria for assessing peripheral arthritis in patients with psoriatic arthritis: analysis of data from randomized, controlled trials of two anti TNF inhibitors. Ann Rheum Dis 65:1373-1378

17. Gladman DD, Mease PJ, Healy P, Fitzgerald O, Cauli A, Lubrano E et al (2007) Outcome measure in psoriatic arthritis. J Rheumatol 34:1159-1166

18. Spadaro A, Ceccarelli F, Scrivo R, Valesini G (2008) Life-table analysis of etanercept with or without methotrexate in patients with psoriatic arthritis. Ann Rheum Dis 67:1650-1651

19. Hyrich KL, Watson KD, Silman AJ, Symmons DP (2006) Predictors of response to anti-TNF-alpha therapy among patients with rheumatoid arthritis: results from the British Society for Rheumatology Biologics Register. Rheumatology 45:1558-1565

20. Olivieri I, de Portu S, Salvarani C, Cauli A, Lubrano E, Spadaro A et al (2008) The psoriatic arthritis cost evaluation study: a cost-ofillness study on tumor necrosis factor inhibitors in psoriatic arthritis patients with inadequate response to conventional therapy. Rheumatology 47:1664-1670 